\title{
Afatinib vs erlotinib for second-line treatment of Chinese patients with advanced squamous cell carcinoma of the lung
}

This article was published in the following Dove Press journal: OncoTargets and Therapy

\author{
Shun Lu, 'Wei Li, ${ }^{2}$ Caicun \\ Zhou, ${ }^{3}$ Cheng-Ping Hu, ${ }^{4}$ \\ Shukui Qin, ${ }^{5}$ Gang Cheng, 6 \\ Jifeng Feng, ${ }^{7}$ Jie Wang, ${ }^{8,9}$ \\ Agnieszka Cseh, ${ }^{10}$ Barbara \\ Peil, ${ }^{\prime}$ Neil Gibson, ${ }^{12}$ Eva \\ Ehrnrooth, ${ }^{13}$ Li Zhang $^{14}$ \\ 'Shanghai Lung Cancer Center, Shanghai \\ Chest Hospital, Shanghai Jiao Tong \\ University, Shanghai, China; ${ }^{2}$ Cancer Center, \\ First Hospital of Jilin University, Changchun, \\ China; ${ }^{3}$ Department of Oncology, Shanghai \\ Pulmonary Hospital, Shanghai, China; \\ ${ }^{4}$ Department of Respiratory Medicine, \\ Xiangya Hospital, Central South University, \\ Changsha, Hunan, China; ${ }^{5}$ People's \\ Liberation Army Cancer Center, Nanjing \\ Bayi Hospital, Nanjing, Jiangsu, China; \\ ${ }^{6}$ Department of Medical Oncology, Beijing \\ Hospital, National Center of Gerontology, \\ Beijing, China; ${ }^{7}$ Department of Internal \\ Medicine, Jiangsu Provincial Tumor Hospital, \\ Nanjing, China; ${ }^{8}$ Department of Internal \\ Medicine, Capital Medical University, \\ Beijing, China; ${ }^{9}$ Peking University School \\ of Oncology, Beijing Cancer Hospital, \\ Beijing, China; ${ }^{10}$ Department of Medical \\ Affairs, Boehringer Ingelheim RCV GmbH \& \\ Co. KG, Vienna, Austria; 'Biostatistics, \\ Boehringer Ingelheim Pharma GmbH \& Co. \\ KG. Ingelheim, Germany; ${ }^{12}$ Translational \\ Medicine and Clinical Oncology, Boehringer \\ Ingelheim Pharma GmbH \& Co. KG. \\ Biberach, Germany; ${ }^{13}$ Division of Oncology, \\ Boehringer Ingelheim, Danmark A/S, \\ Copenhagen, Denmark; ${ }^{14}$ Department of \\ Medical Oncology, Sun Yat-Sen University \\ Cancer Center, Guangzhou, China
}

Correspondence: Shun Lu Shanghai Lung Cancer Center, Shanghai Chest Hospital, Shanghai Jiao Tong University, 24I West Huaihai Road, Xuhui District, Shanghai 200030, China

Tel +86 2l 22200000

Fax +8621 64085875

Email shun_lu@hotmail.com
Background: The global Phase III LUX-Lung 8 trial (ClinicalTrials.gov: NCT01523587) identified significant improvements in progression-free survival (PFS), overall survival (OS), and patient-reported outcomes (PROs) with second-line afatinib vs erlotinib in patients with advanced squamous cell carcinoma (SCC) of the lung.

Materials and methods: We conducted a post hoc analysis of data for patients in LUX-Lung 8 from mainland China $(n=67)$. Compared with erlotinib, afatinib reduced the risk of disease progression or death (PFS) in the Chinese subgroup by 30\% (HR=0.70; 95\% CI: $0.38-1.27$ ).

Results: The risk of death was reduced by $31 \%$ ( $\mathrm{HR}=0.69$; 95\% CI: $0.39-1.21$ ). The proportion of Chinese patients with improvements in PROs also favored afatinib vs erlotinib (global health status/quality of life [QoL], $52.8 \%$ vs $29.6 \%, P=0.072$; dyspnea, $47 \%$ vs $26 \%, P=0.091$; "dyspnea walked", $44 \%$ vs $15 \%, P=0.017$; QoL rate, $53 \%$ vs $26 \%, P=0.037$ ).

Discussion: While this analysis was not powered to demonstrate differences compared to the overall trial population (OTP), and there were some differences in baseline characteristics (eg, the proportion of patients aged $\geq 65$ years old), the benefits of afatinib treatment in Chinese patients with SCC of the lung appeared to be at least comparable to that observed in LUX-Lung 8. As with the OTP, the most common adverse events (AEs) with afatinib in the Chinese subgroup were diarrhea and rash/acne, and the incidence and type of the most frequently occurring AEs were similar. Conclusion: The results suggest that afatinib represents a feasible treatment option for Chinese patients with advanced SCC of the lung following progression on platinum-based chemotherapy. Keywords: afatinib, NSCLC, squamous cell carcinoma, second-line, Phase III, Chinese patients

\section{Introduction}

Lung cancer is the most frequently diagnosed malignancy and leading cause of cancer death in China. ${ }^{1}$ Incidence and mortality rates are increasing steadily, ${ }^{2}$ partly due to the dramatic increase in the prevalence of cigarette smoking in China over the past 30 years, which replicated the US trends of $\sim 40$ years earlier. ${ }^{3-7}$ As a result, further increases in the prevalence of lung cancer and related deaths are predicted..$^{4,8,9}$ In particular, as squamous cell carcinoma (SCC) accounts for a third of lung cancers in China, ${ }^{9}$ increases in the number of cases are anticipated. Chinese patients with non-small-cell lung cancer (NSCLC) differ from Western patients in many ways, including differences in driver mutations, etiologies, and tolerances to treatment. ${ }^{10}$ Despite the growing burden and the need for additional treatment options, recently approved drugs such as the immune checkpoint inhibitors nivolumab, ${ }^{11}$ pembrolizumab, ${ }^{12}$ and atezolizumab, ${ }^{13,14}$ and the anti-VEGFR-2 antibody ramucirumab (in combination with docetaxel), ${ }^{15}$ have yet to be investigated in patients from Asia or China. Treatment options in China for stage 
IV SCC of the lung following progression on platinum-based chemotherapy are limited to single-agent therapy with docetaxel, gemcitabine, vinorelbine, or ifosfamide.

Given that $\sim 60 \%-80 \%$ of SCC tumors express EGFR, ${ }^{16,17}$ and other ErbB family members such as HER2/ErbB2, and ErbB3 are implicated in the pathogenesis of SCC, ${ }^{18}$ there is a clear biological rationale for assessing agents that target ErbB signaling in patients with advanced SCC of the lung. The global LUX-Lung 8 trial evaluated outcomes of secondline treatment with afatinib, an irreversible ErbB family blocker, vs erlotinib, a reversible EGFR tyrosine-kinase inhibitor (TKI), in this setting, following progression after platinum-based chemotherapy. ${ }^{19}$ Significant improvements in progression-free survival (PFS), overall survival (OS), and patient-reported outcomes (PROs) were demonstrated with afatinib vs erlotinib; the safety profile of afatinib was predictable and manageable. ${ }^{19,20}$ On the basis of these findings, afatinib gained global regulatory approval for treatment of locally advanced or metastatic NSCLC of squamous histology and progression during/after platinum-based chemotherapy, and was recently approved in China for the same indication.

Currently, there is a paucity of data on outcomes of afatinib treatment in Chinese patients with advanced SCC of the lung. We conducted a post hoc analysis of patients' data from Chinese study centers in LUX-Lung 8, to evaluate whether treatment outcomes (efficacy and PROs) and the safety of afatinib in Chinese patients were comparable to the overall study population.

\section{Materials and methods}

\section{Patients and study design}

LUX-Lung 8 (ClinicalTrials.gov NCT01523587) was a randomized, controlled, Phase III trial, conducted globally in 183 cancer centers, nine of which were in China. The study design was published previously. ${ }^{19}$ Briefly, eligible patients had a confirmed diagnosis of stage IIIB or IV SCC of the lung, measurable disease according to Response Evaluation Criteria in Solid Tumors (RECIST) version 1.1, Eastern Cooperative Oncology Group performance status of 0 or 1 , and disease progression after $\geq 4$ cycles of platinum-based chemotherapy (Supplementary slides).

Patients were randomized (1:1) to afatinib $(40 \mathrm{mg})$ or erlotinib (150 mg), orally once daily; randomization was stratified by eastern Asian vs non-eastern Asian ethnic origin. The dose of afatinib was individualized according to tolerability. If patients had any grade $\geq 3$ drug-related adverse events (AEs), or grade $\geq 2$ diarrhea lasting 2 days or more, or nausea or vomiting for 7 consecutive days or more despite best supportive care, then afatinib was suspended for $\leq 14$ days. After interruption of treatment and recovery to grade $\leq 1$ or the baseline grade, afatinib was resumed at a lower dose (reduced by $10 \mathrm{mg}$ decrements to a minimum of $20 \mathrm{mg} /$ day). Treatment was permanently discontinued in patients who did not recover to grade $\leq 1$ or the baseline grade. Dose reductions for erlotinib were also permitted. In both arms, treatment was continued until disease progression, unacceptable AEs preventing continuation, or any other reason necessitating withdrawal.

The study was conducted in accordance with the Declaration of Helsinki and guidelines on Good Clinical Practice, and the protocol was approved by local ethics committees at each participating center (Table S1). All patients provided written informed consent for trial participation.

\section{Outcomes and assessments}

The primary endpoint of LUX-Lung $8{ }^{19}$ was PFS, assessed by a blinded central independent review committee, according to RECIST (version 1.1), and the key secondary endpoint was OS. Other secondary endpoints included objective response rate (ORR) and disease control rate (DCR). Patients who received afatinib for 12 months or more were identified (post hoc) as long-term responders (LTRs).

Given the major impact of NSCLC symptoms on quality of life (QoL), ${ }^{21}$ PROs were also evaluated, in particular, global health status (GHS). PROs were assessed at the first visit of each treatment course. The following were evaluated using the European Organisation for the Research and Treatment of Cancer (EORTC) Core Quality of Life Questionnaire-30 (QLQ-C30) and the Quality of Life Questionnaire-lung cancer-specific module (QLQ-LC13): improvements in GHS/QoL (QLQ-C30 questions 29-30); time to deterioration (TTD); and changes in pre-specified lung cancer symptoms over time, ie, cough (QLQ-LC13 question 1), dyspnea (QLQLC13 questions 3-5), and pain (QLQ-C30 questions 9 and 19). ${ }^{22-24}$ PRO responses were converted to a 0-100 scale and analyzed according to EORTC scoring algorithms. ${ }^{22}$ An increase in functional scale or decrease in symptom scale of at least 10 points from baseline for each symptom or category was defined as an improvement, while TTD was defined as the time to a 10-point worsening from the baseline score. AEs were graded using the Common Terminology Criteria for Adverse Events (version 3.0).

All efficacy analyses were undertaken in the randomized intention-to-treat population; descriptive safety analyses included all patients who received $\geq 1$ dose of study medication. The subgroup analysis was conducted to assess whether 
outcomes in Chinese patients and the overall population were similar, although the trial was not powered to detect significant differences in outcomes between subgroups of patients in the afatinib and erlotinib arms.

A Cox proportional-hazards model (stratified by ethnic origin) was used to estimate HRs and 95\% CIs for survival. Treatment groups were compared using a log-rank test. Kaplan-Meier estimates and 95\% CIs were calculated using Greenwood's standard error estimate. Logistic regression models were used to compare proportions of patients with a response, or disease control, between subgroups. Statistical analyses were conducted using SAS version 9.2 or later (SAS Institute Inc., Cary, NC, USA).

\section{Results \\ Patients}

Of 795 patients randomized to treatment in LUX-Lung 8, 67 (8.4\%) were from mainland China; 36 received afatinib and 31 erlotinib. Baseline demographic and clinical characteristics of the Chinese subgroup and the overall LUX-Lung 8 trial population (OTP) are shown in Table 1.
Generally, the baseline characteristics of the Chinese subgroup were similar to the OTP, although fewer Chinese patients were $\geq 65$ years old. In the Chinese subgroup, a greater proportion of females was randomized to afatinib $(13.9 \%)$ than to erlotinib $(6.5 \%)$; the same was true for patients aged $\geq 65$ years ( $36.1 \%$ vs $22.6 \%$ ).

\section{Efficacy outcomes}

At the time of data cut-off, the mean duration of treatment in the Chinese patients was 5.3 months for afatinib (range: 0.5-27.6 months) and 3.5 months for erlotinib (range: 0.3-20.4 months). At the time of primary analysis of survival for the Chinese subgroup (March 2, 2015) median PFS was 2.8 months in both groups (HR=0.70; 95\% CI: 0.38-1.27; Figure 1A; Table 2). Median OS was 10.8 months with afatinib and 8.2 months with erlotinib ( $\mathrm{HR}=0.69 ; 95 \% \mathrm{CI}$ : 0.39-1.21; Figure 1B; Table 2). The HRs for both PFS and OS favored afatinib.

In the OTP, post hoc analysis was undertaken with the aim of identifying possible clinical and biomarkers indicative of long-term response to afatinib. Of 21 LTRs ( $5 \%$ of the OTP),

Table I Patient demographics and baseline characteristics in the Chinese subgroup and the overall LUX-Lung 8 population

\begin{tabular}{|c|c|c|c|c|}
\hline \multirow[t]{2}{*}{ Characteristic } & \multicolumn{2}{|c|}{ Chinese patients $(\mathrm{N}=67)$} & \multicolumn{2}{|c|}{ Overall population $(\mathrm{N}=795)^{\mathrm{a}}$} \\
\hline & Afatinib $(n=36)$ & Erlotinib $(n=3 I)$ & Afatinib $(n=398)$ & Erlotinib $(n=397)$ \\
\hline \multicolumn{5}{|l|}{ Sex, n (\%) } \\
\hline Female & $5(13.9)$ & $2(6.5)$ & $63(15.8)$ & $66(16.6)$ \\
\hline Male & $31(86.1)$ & $29(93.5)$ & $335(84.2)$ & $331(83.4)$ \\
\hline Median age, years (range) & $62.0(36-74)$ & $60.0(43-78)$ & $65.0(36-84)$ & $64.0(35-88)$ \\
\hline$\geq 65$ years, $n(\%)$ & $13(36.1)$ & $7(22.6)$ & $209(52.5)$ & $187(47.1)$ \\
\hline \multicolumn{5}{|l|}{ ECOG PS, n (\%) } \\
\hline 0 & $9(25.0)$ & $8(25.8)$ & $126(31.7)$ & $134(33.8)$ \\
\hline 1 & $27(75.0)$ & $23(74.2)$ & $269(67.6)$ & $262(66.0)$ \\
\hline $2^{\mathrm{b}}$ & 0 & 0 & $3(0.8)$ & $\mathrm{I}(0.3)$ \\
\hline \multicolumn{5}{|l|}{ Ethnic origin, $n(\%)$} \\
\hline Non-eastern Asian & 0 & 0 & $312(78.4)$ & $311(78.3)$ \\
\hline Eastern Asian & $36(100.0)$ & $31(100.0)$ & $86(21.6)$ & $86(21.7)$ \\
\hline \multicolumn{5}{|l|}{ Smoking status, n (\%) } \\
\hline Never smoked & $5(13.9)$ & $5(16.1)$ & $26(6.5)$ & $18(4.5)$ \\
\hline Light ex-smoker ${ }^{c}$ & 0 & $2(6.5)$ & II (2.8) & $12(3.0)$ \\
\hline Other current or ex-smoker ${ }^{d}$ & $31(86.1)$ & $24(77.4)$ & $361(90.7)$ & $367(92.4)$ \\
\hline Median time since diagnosis, years (range) & $0.7(0.3-6.6)$ & $0.6(0.2-6.2)$ & $0.8(0.2-9.3)$ & $0.7(0.2-13.5)$ \\
\hline \multicolumn{5}{|l|}{ Tumor histology, n (\%) } \\
\hline Squamous & $33(91.7)$ & $28(90.3)$ & $381(95.7)$ & $382(96.2)$ \\
\hline Mixed & $3(8.3)$ & $2(6.5)$ & $17(4.3)$ & II (2.8) \\
\hline Undifferentiated ${ }^{e}$ & 0 & $\mathrm{I}(3.2)$ & 0 & $4(1.0)$ \\
\hline \multicolumn{5}{|l|}{ Clinical stage at screening, n (\%) } \\
\hline IIIA & $\mathrm{I}(2.8)$ & 0 & $\mathrm{I}(0.3)$ & $4(1.0)$ \\
\hline IIIB & $7(19.4)$ & $5(16.1)$ & $48(12.1)$ & $48(12.1)$ \\
\hline IV & $28(77.8)$ & $26(83.9)$ & $349(87.7)$ & $345(86.9)$ \\
\hline
\end{tabular}

Notes: aReprinted from Lancet Oncol. 2015;16(8). Soria JC, Felip E, Cobo M, et al. Afatinib versus erlotinib as second-line treatment of patients with advanced squamous cell carcinoma of the lung (LUX-Lung 8): an open-label randomised controlled phase 3 trial. 897-907, Copyright (2015), with permission from Elsevier; 19 bProtocol violations; $c<15$ pack-years and stopped $>$ I year before diagnosis; ${ }^{c}$ in the overall population, 7 I (I7.8\%) patients treated with afatinib vs 85 (2I.4\%) treated with erlotinib were current smokers; in the Chinese population, four (1 I.1\%) vs three (9.7\%) patients were current smokers; ${ }^{e}$ originally classed as squamous by the treating investigator.

Abbreviation: ECOG PS, Eastern Cooperative Oncology Group performance status. 


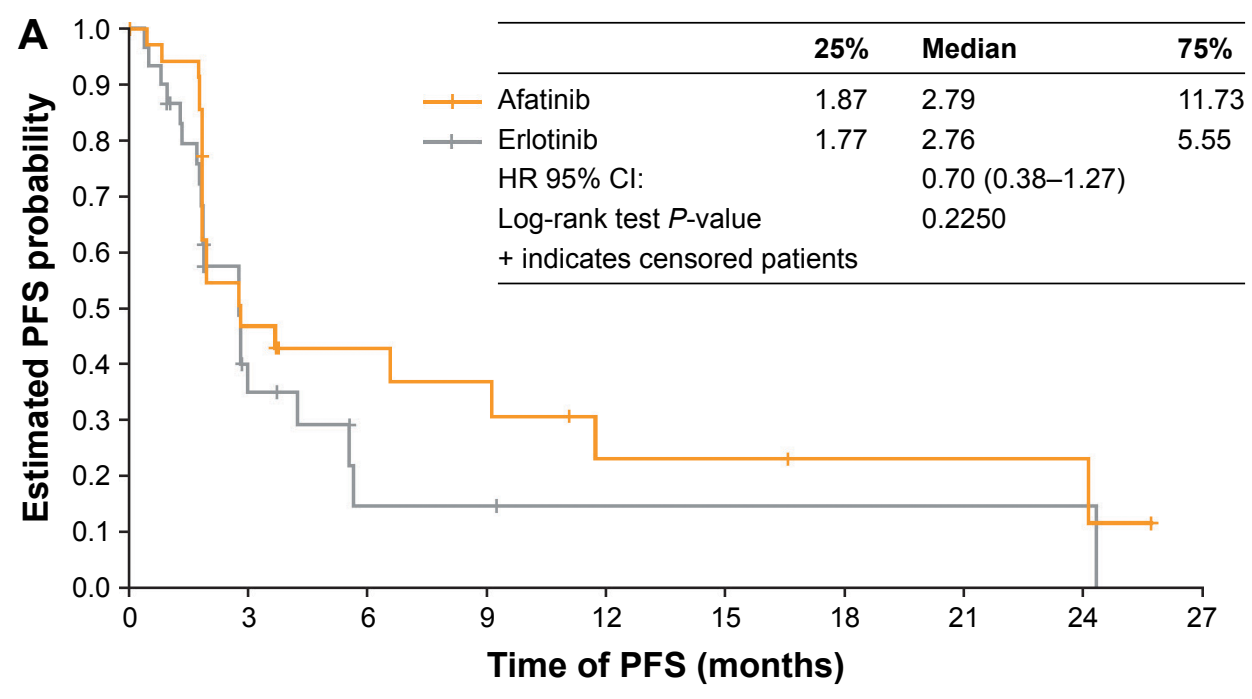

$\begin{array}{lllllllllll}\text { Number at risk: } & & & & & & & \\ \text { Afatinib } & 36 & 12 & 7 & 6 & 3 & 3 & 2 & 2 & 2 & 0 \\ \text { Erlotinib } & 31 & 7 & 2 & 2 & 1 & 1 & 1 & 1 & 1 & 0\end{array}$

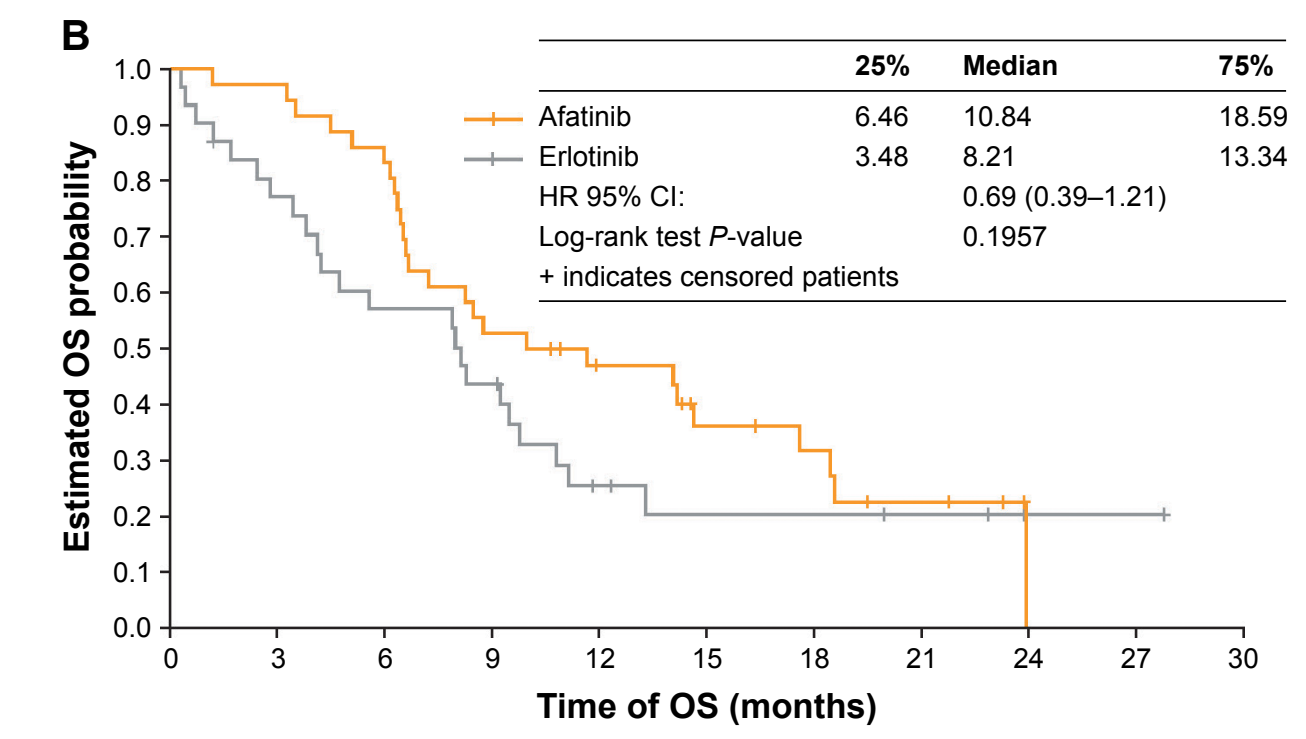

Number at risk:

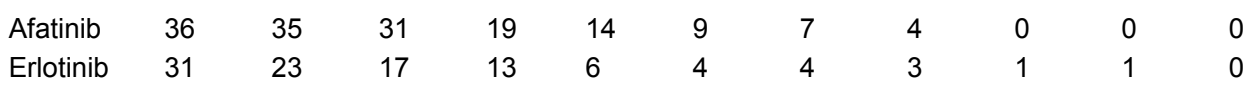

Figure I (A) PFS (independent review) and (B) OS for the Chinese subgroup. Abbreviations: OS, overall survival; PFS, progression-free survival.

four (11\%) were from the Chinese subgroup. The median duration of treatment in these patients was 22.7 months (range: 16.6-27.6 months). At data cut-off, three of the four Chinese LTRs ( $8 \%$ ) were alive and still on treatment (their OS was 17.5 months, 26.6 months, and 26.8 months; Figure 2 and Supplementary slides), while the fourth had disease progression (OS 17.6 months; Figure 2 and Supplementary slides). Two of the four LTRs had a confirmed objective response (OR); two had partial responses (including one female neversmoker with mixed squamous histology) and two had stable non-target disease in the absence of baseline target disease (non-complete response/non-progressive disease [NN]; Figure 2 and Supplementary slides). One Chinese patient randomized to erlotinib had been treated for 20.3 months, with a best response of $\mathrm{NN}$ and OS of 24.3 months.

ORRs in the Chinese subgroup were $8.3 \%$ for afatinib and $6.5 \%$ for erlotinib ( $P=0.772$; Table 2$)$. DCRs were $55.6 \%$ and $41.9 \%$ respectively ( $P=0.271$; Table 2$)$. Changes in target lesion sum of diameters from baseline are shown in Figure S1 and Supplementary slides. 
Table 2 PFS by independent review, OS, and best overall tumor response by independent review, in the Chinese subgroup and the overall LUX-Lung 8 population

\begin{tabular}{|c|c|c|c|c|c|c|c|c|}
\hline \multirow[t]{2}{*}{ Parameter } & \multicolumn{4}{|c|}{ Chinese subgroup $(\mathrm{N}=67)$} & \multicolumn{4}{|c|}{ Overall population $(\mathrm{N}=795)^{\mathrm{a}}$} \\
\hline & $\begin{array}{l}\text { Afatinib } \\
(n=36)\end{array}$ & $\begin{array}{l}\text { Erlotinib } \\
(n=31)\end{array}$ & HR $(95 \% \mathrm{Cl})$ & $P$-value & $\begin{array}{l}\text { Afatinib } \\
(n=398)\end{array}$ & $\begin{array}{l}\text { Erlotinib } \\
(n=397)\end{array}$ & HR (95\% Cl) & $P$-value \\
\hline Median PFS, months & 2.8 & 2.8 & $0.70(0.38-1.27)$ & 0.2250 & 2.4 & 1.9 & $0.82(0.68-1.00)$ & 0.0427 \\
\hline Median OS, months & 10.8 & 8.2 & $0.69(0.39-1.2 I)$ & 0.1957 & 7.9 & 6.8 & $0.81(0.69-0.95)$ & 0.0077 \\
\hline Disease control, n (\%) & $20(55.6)$ & $13(4 \mid .9)$ & & 0.2713 & $201(50.5)$ & $157(39.5)$ & & 0.0020 \\
\hline Objective response, $\mathrm{n}(\%)$ & $3(8.3)$ & $2(6.5)$ & & 0.7723 & $22(5.5)$ & II (2.8) & & 0.0551 \\
\hline Complete response, $\mathrm{n}(\%)$ & 0 & 0 & & NA & I $(0.3)$ & $0(0)$ & & NA \\
\hline Partial response, n (\%) & $3(8.3)$ & $2(6.5)$ & & NA & $21(5.3)$ & II (2.8) & & NA \\
\hline Stable disease, n (\%) & $9(25.0)$ & $7(22.6)$ & & NA & $124(3 \mid .2)$ & $103(25.9)$ & & NA \\
\hline $\begin{array}{l}\text { Non-complete response/ } \\
\text { non-progressive disease, }{ }^{\mathrm{b}} \mathrm{n}(\%)\end{array}$ & $8(22.2)$ & $4(12.9)$ & & NA & $55(13.8)$ & $43(10.8)$ & & NA \\
\hline Progressive disease, n (\%) & $16(44.4)$ & $8(25.8)$ & & NA & I 33 (33.4) & $169(42.6)$ & & NA \\
\hline Not evaluable, n (\%) & 0 & $10(32.3)$ & & NA & $64(16.1)$ & $7 \mid(17.9)$ & & NA \\
\hline
\end{tabular}

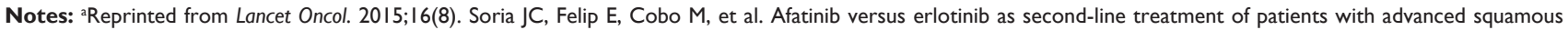
cell carcinoma of the lung (LUX-Lung 8): an open-label randomised controlled phase 3 trial. 897-907, Copyright (2015), with permission from Elsevier; ${ }^{19}$ bStable non-target disease in the absence of baseline target disease.

Abbreviations: NA, not applicable; OS, overall survival; PFS, progression-free survival.

\section{PROs}

Among the Chinese subgroup, $52.8 \%$ of patients had an improvement in GHS/QoL with afatinib, vs $29.6 \%$ with erlotinib ( $P=0.072$; Table S1). Improvements in individual PROs also occurred, including dyspnea (in $47 \%$ of patients taking afatinib vs $26 \%$ taking erlotinib; $P=0.091$ ), with significant differences in favor of afatinib in: "dyspnea walked" (afatinib, 44\%; erlotinib, 15\%; $P=0.017$ ); and QoL rate (afatinib, 53\%; erlotinib, 26\%; $P=0.037$; Table S1). TTD of cough was significantly longer for afatinib (median 4.9 months) than for erlotinib ( 2.9 months; HR $=0.46 ; 95 \% \mathrm{CI}$ : $0.22-0.94 ; P=0.03$; Figure 3 ).

\section{Safety outcomes}

The most frequently occurring AEs (any grade, and grade 3 ) in the Chinese subgroup and the overall study population are shown in Table 3; the most common AEs with afatinib in both the overall study population and the Chinese subgroup were diarrhea and rash/acne. The incidences of most AEs were similar between the overall population and the Chinese subgroup; notable discrepancies (of $>10$ percentage points) were for fatigue (34\% and $19 \%$, respectively), decreased appetite ( $25 \%$ and $6 \%)$, nausea ( $21 \%$ and $8 \%$ ), and vomiting (13\% and $0 \%)$. Overall, grade 3 AEs occurred in $22 \%$ of patients in the Chinese afatinib group (8/36; Table 3$)$, and serious AEs (SAEs) occurred in 22\% (8/36; Table S2). The most frequently occurring AEs with erlotinib in both the Chinese subgroup and the overall study population were rash/ acne, fatigue, and diarrhea, each of which was more frequent in the overall population than the Chinese subgroup (rash/ acne, $70 \%$ and $52 \%$; fatigue, $30 \%$ and $19 \%$; diarrhea, $41 \%$ and $13 \%$; Table 3). Grade 3 AEs occurred in $19 \%$ of the

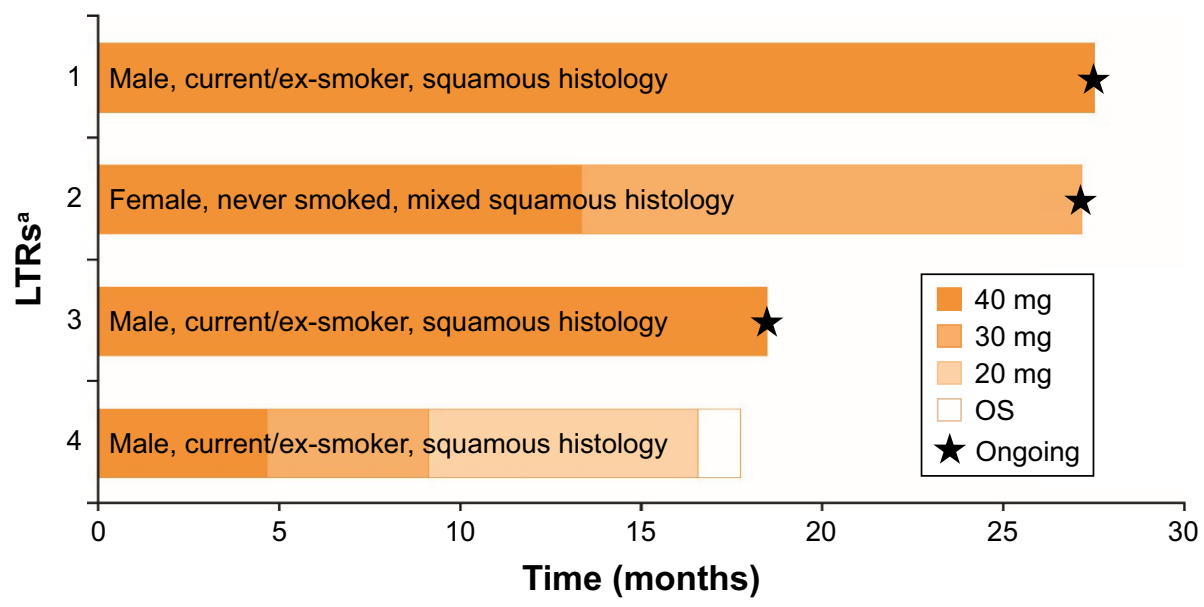

Figure 2 Long-term benefit of afatinib in LTRs (Chinese patients).

Notes: a Patients ordered by treatment duration. Values are duration of treatment at the doses indicated.

Abbreviations: LTRs, long-term responders; OS, overall survival. 


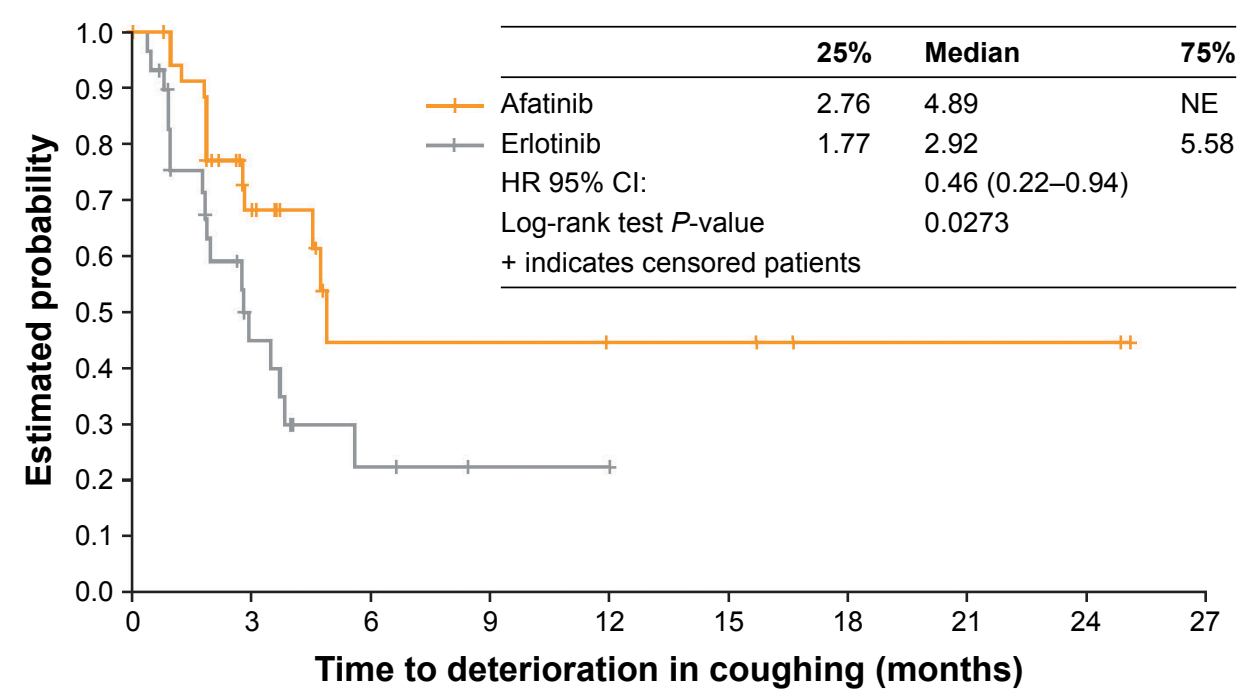

\section{Number at risk:}

$\begin{array}{lllllllllll}\text { Afatinib } & 36 & 14 & 5 & 5 & 4 & 4 & 2 & 2 & 2 & 0 \\ \text { Erlotinib } & 31 & 9 & 3 & 1 & 0 & 0 & 0 & 0 & 0 & 0\end{array}$

Figure 3 Time to deterioration in coughing in the Chinese subgroup. Abbreviation: NE, not evaluable.

Chinese erlotinib group (6/31; Table 3) and SAEs occurred in $36 \%(11 / 31$; Table S2).

Five Chinese patients (14\%), including two LTRs, required a dose reduction due to AEs while taking afatinib (within 1 month of initiating treatment in three of the five patients), and four (11\%) discontinued due to an $\mathrm{AE}$ (Table S3). Five Chinese patients in the afatinib arm died during the study (14\%). The causes were lung infection (one patient), progression of malignant neoplasm (three patients), and multi-organ failure (one patient); none was considered related to treatment by the investigators.

\section{Discussion}

In LUX-Lung 8, afatinib significantly improved PFS, OS, and PROs (vs erlotinib) in patients with advanced SCC of the lung after failure of platinum-based therapy, while the safety profile of afatinib was predictable and manageable. In this post hoc analysis, we demonstrated trends toward improved

Table $3 A E^{a} s$ in the Chinese subgroup and the overall safety population

\begin{tabular}{|c|c|c|c|c|c|c|c|c|}
\hline \multirow{3}{*}{$\begin{array}{l}\text { Patients with } \\
\text { AE }^{\mathrm{a}} \text { s, n (\%) }\end{array}$} & \multicolumn{4}{|c|}{ Chinese subgroup $(\mathrm{N}=67)$} & \multicolumn{4}{|c|}{ Overall safety population $(\mathrm{N}=787)^{\mathrm{b}}$} \\
\hline & \multicolumn{2}{|c|}{ Afatinib $(n=36)$} & \multicolumn{2}{|c|}{ Erlotinib $(n=31)$} & \multicolumn{2}{|c|}{ Afatinib $(n=392)$} & \multicolumn{2}{|c|}{ Erlotinib $(n=395)$} \\
\hline & All & Grade 3 & All & Grade 3 & All & Grade 3 & All & Grade 3 \\
\hline Any AE & $36(100.0)$ & $8(22.2)$ & $28(90.3)$ & $6(19.4)$ & $390(99.5)$ & $124(3 \mid .6)$ & $385(97.5)$ & 138 (34.9) \\
\hline Diarrhea & $27(75.0)$ & 0 & $4(12.9)$ & 0 & $293(74.7)$ & $39(9.9)$ & $163(41.3)$ & $12(3.0)$ \\
\hline Rash/acne ${ }^{c}$ & $23(63.9)$ & I (2.8) & $16(5 \mid .6)$ & 0 & $273(69.6)$ & $26(6.6)$ & $276(69.9)$ & $42(10.6)$ \\
\hline Stomatitis ${ }^{c}$ & $14(38.9)$ & $2(5.6)$ & $2(6.5)$ & 0 & $118(30.1)$ & $16(4.1)$ & $42(10.6)$ & $2(0.5)$ \\
\hline Fatigue $^{c}$ & $7(19.4)$ & 0 & $6(19.4)$ & I (3.2) & $132(33.7)$ & $19(4.8)$ & $119(30.1)$ & $23(5.8)$ \\
\hline Hemoptysis & $7(19.4)$ & 0 & $3(9.7)$ & 0 & $49(12.5)$ & $2(0.5)$ & $49(12.4)$ & $2(0.5)$ \\
\hline Dyspnea & $6(16.7)$ & $\mathrm{I}(2.8)$ & I (3.2) & 0 & $79(20.2)$ & $12(3.1)$ & $94(23.8)$ & $18(4.6)$ \\
\hline Hypokalemia & $6(16.7)$ & I (2.8) & I (3.2) & 0 & $20(5.1)$ & $9(2.3)$ & $12(3.0)$ & $3(0.8)$ \\
\hline Cough & $4(I I . I)$ & 0 & $3(9.7)$ & $\mathrm{I}(3.2)$ & $65(16.6)$ & $2(0.5)$ & $69(17.5)$ & $2(0.5)$ \\
\hline Paronychia $^{c}$ & $4(I I . I)$ & 0 & 0 & 0 & $43(11.0)$ & $2(0.5)$ & $20(5.1)$ & I $(0.3)$ \\
\hline Decreased appetite & $2(5.6)$ & $\mathrm{I}(2.8)$ & $3(9.7)$ & $\mathrm{I}(3.2)$ & 97 (24.7) & $12(3.1)$ & $103(26.1)$ & $8(2.0)$ \\
\hline Nausea & $3(8.3)$ & 0 & $2(6.5)$ & I (3.2) & $81(20.7)$ & $6(1.5)$ & $64(16.2)$ & $4(1.0)$ \\
\hline Vomiting & 0 & 0 & $2(6.5)$ & 0 & $51(13.0)$ & $3(0.8)$ & $40(10.1)$ & $4(1.0)$ \\
\hline Constipation & $2(5.6)$ & 0 & $\mathrm{I}(3.2)$ & 0 & $43(11.0)$ & 0 & $43(10.9)$ & $\mathrm{I}(0.3)$ \\
\hline
\end{tabular}

Notes: aThe data shown are for AEs that occurred (at any grade) in $>10 \%$ of patients in the afatinib arm, in either the overall population or the Chinese subgroup. AEs were coded using MedDRA version 17.I. Of the AEs listed, the following also occurred at grade $4 / 5$ in the afatinib arm (overall safety population): grade 4 diarrhea ( $n=3$ ); grade 4 fatigue $(n=1)$; grade 5 fatigue $(n=1)$; grade 4 dyspnea $(n=3)$; grade 5 dyspnea $(n=3)$; grade 5 hemoptysis $(n=2)$. b ${ }^{\text {bata }}$ on file; 'grouped term.

Abbreviations: AE, adverse event; MedDRA, Medical Dictionary for Regulatory Activities. 
PFS and OS with afatinib vs erlotinib, and improvements in PROs, in Chinese patients. This analysis was not powered to demonstrate differences compared with the OTP and there were several differences in baseline characteristics between the Chinese subset and the OTP (eg, more afatinib-treated patients in the OTP were $\geq 65$ years old than in the Chinese subset). Nevertheless, the results of the current analysis indicated that the outcomes of afatinib treatment in Chinese patients were consistent with LUX-Lung 8 (Table 2). ${ }^{19}$ Although the outcomes were consistent with the OTP, the CIs for the PFS and OS HRs overlap unity, due to the small size of the Chinese subgroup.

Four of the 36 Chinese patients (11\%) were LTRs to afatinib (only one of 31 Chinese patients randomized to erlotinib was an LTR). Two of the four Chinese LTRs to afatinib had a confirmed OR; the median duration of treatment was 22.7 months and 17.6 months, respectively. Three of the Chinese LTRs were still receiving treatment at the time of data cut-off. Although not directly comparable, these data appear to be consistent with the OTP. Twenty-one patients $(5 \%)$ treated with afatinib in the OTP were LTRs, seven of whom had a confirmed OR. ${ }^{25}$

Impact on patients' health-related QoL is a major consideration when considering the evidence for different oral anticancer drugs. In a survey of 83 patients with advanced lung cancer, one third rated QoL as more important than length of life, and more than half rated the two as equally important. ${ }^{26}$ In the current analysis, $53 \%$ of Chinese patients treated with afatinib had an improvement in GHS/QoL compared with $30 \%$ of erlotinib-treated patients. These data are at least comparable to the OTP ( $36 \%$ vs $28 \%$, respectively; $P=0.041),{ }^{20}$ but did not achieve statistical significance $(P=0.072)$, probably due to the relatively small sample size. In the Chinese subgroup, PROs that improved with afatinib included GHS/ QoL, "dyspnea walked", and TTD of cough. Lung cancer symptoms (dyspnea, pain, and fatigue) can interfere with activities of daily life, even when less severe. ${ }^{21}$ In the Chinese subgroup, afatinib improved PROs related to dyspnea and cough, and improved GHS/QoL and QoL rate.

While EGFR TKIs are generally better tolerated than chemotherapy, some AEs (particularly gastrointestinal and cutaneous AEs) occur relatively frequently. ${ }^{27}$ Indeed, in LUXLung 8 , diarrhea of any grade occurred in $75 \%$ of the afatinib group, and rash/acne in 70\%; incidences in the Chinese subgroup were $75 \%$ and $64 \%$, respectively. However, none of the Chinese patients discontinued afatinib due to diarrhea or rash/acne, suggesting that clinicians in China were successful in managing the impact of AEs, possibly by means of tolerability-guided afatinib dose adjustments. The incidence and type of the most frequently occurring AEs were otherwise similar between the Chinese subgroup and the OTP (Table 3).

There were no instances of grade $\geq 3$ diarrhea or discontinuations due to diarrhea in the Chinese subgroup treated with afatinib; the frequency of SAEs was $22 \%$. The frequency of dose reductions and dose discontinuations due to AEs in Chinese patients treated with afatinib were $14 \%$ and $11 \%$, respectively.

Given that EGFR mutations in SCC of the lung are rare, ${ }^{28,29}$ $E G F R$ mutation testing was not mandated, and consequently, EGFR mutation data were not available for the Chinese subgroup, although next-generation sequencing was conducted in 238 patients ( $~ 30 \%$ of the overall LUX-Lung 8 population), retrospectively selected according to clinical benefit with afatinib or erlotinib (PFS $>2$ months, $n=144$; PFS $\leq 2$ months, $\mathrm{n}=94)$. The proportion of patients with EGFR mutation was low overall $(\mathrm{n}=14[6 \%]),{ }^{19}$ and it is therefore unlikely that the improved survival outcomes detected with afatinib were driven by molecular aberrations of EGFR ${ }^{19}$ Given the role of HER2 and ErbB3 in the pathogenesis of SCC (overexpression occurs in $20 \%-30 \%$ of squamous tumors), ${ }^{18}$ and the involvement of several signaling molecules downstream of the ErbB receptors, broader irreversible ErbB blockade with afatinib may inactivate aberrant ErbB-dependent signaling cascades, which may underlie its efficacy in SCC of the lung. ${ }^{19}$

In LUX-Lung 8, compared with erlotinib, afatinib significantly improved PFS, OS, and some PROs relating to symptoms commonly associated with NSCLC. The benefit of afatinib was also apparent in the Chinese subgroup. The availability of multiple tablet strengths of afatinib facilitates simple and effective tolerability-guided dose adjustment in the outpatient setting. ${ }^{30-32}$

\section{Conclusion}

Although this exploratory post hoc subgroup analysis was not powered to demonstrate significant differences between the Chinese subgroup and overall study population, given the need for new approved and convenient treatment options, the results suggest that afatinib represents a suitable treatment option for Chinese patients with advanced SCC of the lung, following progression during or after platinum-based chemotherapy.

\section{Acknowledgments}

This study was funded by Boehringer Ingelheim. Medical writing assistance, supported financially by Boehringer Ingelheim, was provided by Michael Simpson PhD, CMPP of GeoMed, an Ashfield company, part of UDG Healthcare plc, during the preparation of this manuscript. 
We thank the patients, their families, and all the investigators who participated in this study. We acknowledge the support of Claudia Buehnemann of Boehringer Ingelheim Pharma GmbH \& Co. KG in generating the EGFR data.

\section{Author contributions}

All authors contributed to data analysis, drafting and revising the article, gave final approval of the version to be published, and agree to be accountable for all aspects of the work.

\section{Disclosure}

CZ reports lecture fees from AstraZeneca, Boehringer Ingelheim, Eli Lilly, Roche, and Sanofi. AC, BP, NG, and EE report employment by Boehringer Ingelheim. The authors report no other conflicts of interest in this work.

\section{References}

1. Chen W, Zheng R, Zeng H, Zhang S. The incidence and mortality of major cancers in China, 2012. Chin J Cancer. 2016;35(1):73.

2. Hong QY, Wu GM, Qian GS, et al. Prevention and management of lung cancer in China. Cancer. 2015;121 Suppl 17:3080-3088.

3. Li Q, Hsia J, Yang G. Prevalence of smoking in China in 2010. N Engl J Med. 2011;364(25):2469-2470.

4. Molina JR, Yang P, Cassivi SD, Schild SE, Adjei AA. Non-small cell lung cancer: epidemiology, risk factors, treatment, and survivorship. Mayo Clin Proc. 2008;83(5):584-594.

5. Yang L, Parkin DM, Ferlay J, Li L, Chen Y. Estimates of cancer incidence in China for 2000 and projections for 2005. Cancer Epidemiol Biomarkers Prev. 2005;14(1):243-250.

6. Youlden DR, Cramb SM, Baade PD. The International Epidemiology of Lung Cancer: geographical distribution and secular trends. J Thorac Oncol. 2008;3(8):819-831.

7. Zhang H, Cai B. The impact of tobacco on lung health in China. Respirology. 2003;8(1):17-21.

8. Chen W, Zheng R, Zeng H, Zhang S. Epidemiology of lung cancer in China. Thorac Cancer. 2015;6(2):209-215.

9. Gao Y, Zhang JF, Li QC, et al. The clinicopathological and prognostic features of Chinese and Japanese inpatients with lung cancer. Oncotarget. 2016;7(41):67425-67434.

10. Zhou Q, Wu YL. Developing CSCO lung cancer practice guidelines stratified by resource availability and treatment value. J Glob Oncol. 2016;3(4):285-288.

11. Brahmer J, Reckamp KL, Baas P, et al. Nivolumab versus docetaxel in advanced squamous-cell non-small-cell lung cancer. $N$ Engl J Med. 2015;373(2):123-135.

12. Herbst RS, Baas P, Kim DW, et al. Pembrolizumab versus docetaxel for previously treated, PD-L1-positive, advanced non-small-cell lung cancer (KEYNOTE-010): a randomised controlled trial. Lancet. 2016; 387(10027):1540-1550.

13. Fehrenbacher L, Spira A, Ballinger M, et al. Atezolizumab versus docetaxel for patients with previously treated non-small-cell lung cancer (POPLAR): a multicentre, open-label, phase 2 randomised controlled trial. Lancet. 2016;387(10030):1837-1846.

14. Rittmeyer A, Barlesi F, Waterkamp D, et al. Atezolizumab versus docetaxel in patients with previously treated non-small-cell lung cancer (OAK): a phase 3, open-label, multicentre randomised controlled trial. Lancet. 2017;389(10066):255-265.
15. Garon EB, Ciuleanu TE, Arrieta O, et al. Ramucirumab plus docetaxel versus placebo plus docetaxel for second-line treatment of stage IV non-small-cell lung cancer after disease progression on platinum-based therapy (REVEL): a multicentre, double-blind, randomised phase 3 trial. Lancet. 2014;384(9944):665-673.

16. Hirsch FR, Varella-Garcia M, Bunn PA Jr, et al. Epidermal growth factor receptor in non-small-cell lung carcinomas: correlation between gene copy number and protein expression and impact on prognosis. J Clin Oncol. 2003;21(20):3798-3807.

17. Zugazagoitia J, Ponce S, Paz-Ares L. Necitumumab for first-line treatment of advanced, squamous, non-small-cell lung cancer: a relevant step forward? Transl Lung Cancer Res. 2016;5(1):95-97.

18. Hirsh V. New developments in the treatment of advanced squamous cell lung cancer: focus on afatinib. Onco Targets Ther. 2017;10:2513-2526.

19. Soria JC, Felip E, Cobo M, et al. Afatinib versus erlotinib as secondline treatment of patients with advanced squamous cell carcinoma of the lung (LUX-Lung 8): an open-label randomised controlled phase 3 trial. Lancet Oncol. 2015;16(8):897-907.

20. Felip E, Hirsh V, Popat S, et al. Symptom and quality of life improvement in LUX-Lung 8, an open-label Phase III study of second-line afatinib versus erlotinib in patients with advanced squamous cell carcinoma of the lung after first-line platinum-based chemotherapy. Clin Lung Cancer. 2018;19(1):74.e11-83.e11.

21. Tanaka K, Akechi T, Okuyama T, Nishiwaki Y, Uchitomi Y. Impact of dyspnea, pain, and fatigue on daily life activities in ambulatory patients with advanced lung cancer. J Pain Symptom Manage. 2002;23(5):417-423.

22. Aaronson NK, Ahmedzai S, Bergman B, et al. The European Organisation for Research and Treatment of Cancer QLQ-C30: a quality-of-life instrument for use in international clinical trials in oncology. $J$ Natl Cancer Inst. 1993;85(5):365-376.

23. Bergman B, Aaronson NK, Ahmedzai S, Kaasa S, Sullivan M. The EORTC QLQ-LC13: a modular supplement to the EORTC Core Quality of Life Questionnaire (QLQ-C30) for use in lung cancer clinical trials. EORTC Study Group on Quality of Life. Eur J Cancer. 1994;30A(5):635-642.

24. European Organisation for Research and Treatment of Cancer. The EORTC QLQ-C30 scoring manual. European Organisation for Research and Treatment of Cancer; 2001. Available from: http://www.eortc.be/ qol/files/SCManualQLQ-c30.pdf. Accessed June 4, 2018.

25. Gadgeel SM, Soria JC, Felip E, et al. Second-line afatinib vs erlotinib for patients with squamous cell carcinoma (SCC) of the lung (LUXLung 8 [LL8]): analysis of tumour and serum biomarkers and long-term responders. Eur J Cancer. 2017;72(1):S185.

26. Meropol NJ, Egleston BL, Buzaglo JS, et al. Cancer patient preferences for quality and length of life. Cancer. 2008;113(12):3459-3466.

27. Yang JC, Reguart N, Barinoff J, et al. Diarrhea associated with afatinib: an oral ErbB family blocker. Expert Rev Anticancer Ther. 2013; 13(6):729-736.

28. Dearden S, Stevens J, Wu YL, Blowers D. Mutation incidence and coincidence in non small-cell lung cancer: meta-analyses by ethnicity and histology (mutMap). Ann Oncol. 2013;24(9):2371-2376.

29. Cancer Genome Atlas Research Network. Comprehensive genomic characterization of squamous cell lung cancers. Nature. 2012;489(7417): 519-525.

30. Boehringer Ingelheim International GmbH. Gilotrif US Prescribing Information. 2016; Available from: http://docs.boehringer-ingelheim. com/Prescribing\%20Information/PIs/Gilotrif/Gilotrif.pdf?DMW_ FORMAT=pdfGiotrif. Accessed February 13, 2017.

31. GIOTRIF ${ }^{\circledR}$ (afatinib) tablets, for oral use [prescribing information]. Boehringer Ingelheim International $\mathrm{GmbH} ; 2016$.

32. Yang JC, Sequist LV, Zhou C, et al. Effect of dose adjustment on the safety and efficacy of afatinib for EGFR mutation-positive lung adenocarcinoma: post hoc analyses of the randomized LUX-Lung 3 and 6 trials. Ann Oncol. 2016;27(11):2103-2110 


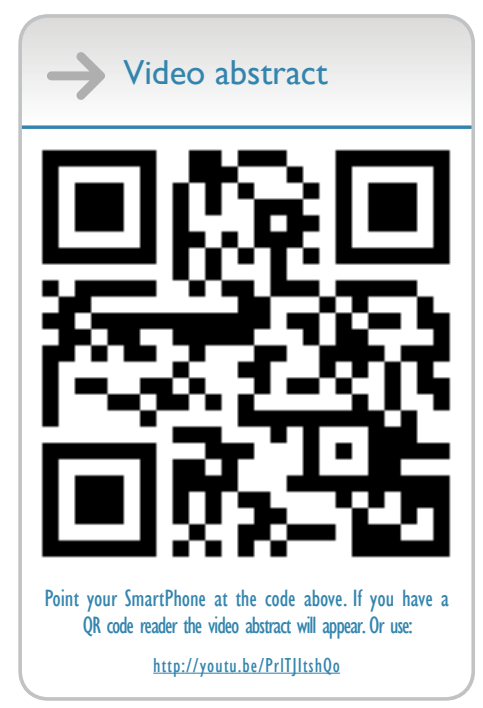

OncoTargets and Therapy

Dovepress

\section{Publish your work in this journal}

OncoTargets and Therapy is an international, peer-reviewed, open access journal focusing on the pathological basis of all cancers, potential targets for therapy and treatment protocols employed to improve the management of cancer patients. The journal also focuses on the impact of management programs and new therapeutic agents and protocols on patient perspectives such as quality of life, adherence and satisfaction. The manuscript management system is completely online and includes a very quick and fair peer-review system, which is all easy to use. Visit http://www.dovepress.com/testimonials.php to read real quotes from published authors. 